\title{
Research and Application of Soil Solidifying Agent
}

\author{
Zhang Yuping ${ }^{1, *}$, WangZilong ${ }^{1}$, Zheng Yifeng $^{2}$ \\ ${ }^{1}$ City College, Southwest University of Science and Technology, Mianyang 621000 \\ ${ }^{2}$ Shanghai Tunnel Engineering Co Ltd, Shanghai 200000
}

\begin{abstract}
The curing mechanism and research status of inorganic soil solidifying agent, organic soil solidifying agent, ionic soil solidifying agent, bioenzyme soil solidifying agent and compound soil solidifying agent were reviewed, and the application of soil solidifying agent in water conservancy and road engineering was introduced. Several suggestions are put forward for the problems existing in soil stabilizer.
\end{abstract}

\section{Introduction}

Soil solidifiers are a kind of material that can be added to the soil at the normal temperature to bind soil particles, or to greatly improve the compactness, strength, elasticity and water resistance of the soil through the ionic bonding, hydration reaction, and biological action with clay minerals. As a new type of building material, soil solidifiers can improve and solidify a large amount of soil, and the treated soil can be used in the foundation building, roadbed construction and other engineering projects. At present, soil solidifiers have been widely used in housing construction, road construction, hydraulic engineering, agricultural engineering, bridge engineering and tunnel engineering.

In 1824, English Inventor Joe Aspidin invented Portland cement, on which basis the cement soil continued to be promoted. However, the cement soil is low in early-stage strength and large in shrinkage degree. In addition, the quality and solidifying effect of soils vary greatly in different regions. For soils with higher organic matter or higher moisture content, cement is less effective in solidification. Therefore, many scholars at home and abroad have begun to expand the choices of raw materials of solidifiers, and develop solidifiers suitable for different soils using various combinations and formulations. Nowadays, there are at least hundreds of solidifiers. The research focus was shifting from the soil solidification in a certain area at the beginning, to the adjustment based on transportation and construction methods, and now to the soil heavy metal lockup and the reuse of industrial residue. The study focus on soil solidifiers has also shifted from simple engineering application to a multidisciplinary and multifunctional soil improvement. Currently, soil solidifiers are mainly divided into four categories, namely, inorganic soil solidifier, organic soil solidifier, ionic soil solidifier and biological enzyme soil solidifier ${ }^{[1]}$.

\section{Solidification mechanism and research status of soil solidifier}

\subsection{Inorganic soil solidifier}

Inorganic soil solidifier is currently the most commonly used soil solidifier. It is usually in the form of powders and is composed of the main solidifying agent and the auxiliary agent. The former is composed of cement, lime, gypsum, fly ash and other industrial residues. Due to the low cost, the wide range of sources (some industrial residues can be used), and its wide applications, inorganic solidifier has been extensively used. However, the main bonding materials of this soil solidifier are cement and lime, which have strong alkalinity, thus causing the improved soil to be unsuitable for growing plants. At the same time, after long-term use, the alkaline matters in the solidifier gradually diffuse with water, affecting the surrounding soil body. In addition, the soil strength after the treatment of inorganic soil solidifier develops slowly. Therefore, inorganic soil solidifiers are usually used together with some inorganic or organic additives so as to eliminate the negative effects brought by the alkaline bonding materials and accelerate the strength development of the solidified soil.

The solidification mechanism of inorganic soil solidifiers mainly includes physical action, chemical action, and physicochemical action ${ }^{[2]}$. The three actions interact with each other, making the soil particles in close contact under the action of the molding pressure. The binding material in the solidifier itself undergoes a hydration reaction, generating calcium silicate hydrate and calcium aluminate hydrate. These hydration products overlap and intersect each other to bond the soil particles together, and the generated amorphous gel fill soil particle pores, further increasing the soil compactness. At the same time, a large amount of $\mathrm{OH}^{-}$will be generated during the hydration reaction of the solidifier material, and $\mathrm{Ca}(\mathrm{OH})_{2}$ will have a volcanic ash effect with the

*Zhang Yuping: zypswust@126.com 
active $\mathrm{SiO}_{2}$ and $\mathrm{Al}_{2} \mathrm{O}_{3}$ in the soil, generating products with gelling properties, further increasing the coagulation effect and improving the soil strength.

As the chemical reactions between the solidifier and the soil continue, a large amount of $\mathrm{Ca}^{2+}$ and $\mathrm{Al}^{3+}$ ions will accumulate in the pores of the solidified soil. These free ions will exchange ions with the adsorbed sodium ions, thereby reducing the thickness of the double electrode layer between soil particles and promoting the agglomeration of soil particles.

Scholars at home and abroad have conducted a lot of research on the role of auxiliary agents and the solidifying mechanism of inorganic solidifiers, which is consistent with the current solidifying mechanism to a certain extent.

$\mathrm{Hu}$ and $\mathrm{Fu}{ }^{[3]}$ studied the effect of inorganic soil solidifiers on the strength, water resistance and frost resistance of raw soil materials and their solidifying mechanisms. The results show that when the content of inorganic soil solidifier is $20 \% \sim 25 \%$, the solidified soil has higher strength, water resistance and frost resistance. They also proposed that the main reason is that the inorganic soil solidifier reacts with $\mathrm{SiO}_{2}$ and $\mathrm{Al}_{2} \mathrm{O}_{3}$ in the raw soil to generate new products such as $\mathrm{Ca} \cdot 1.7 \mathrm{MgO} \cdot 3 \mathrm{SiO}_{4}, 2 \mathrm{CaO} \cdot \mathrm{Al}_{2} \mathrm{O}_{3} \cdot \mathrm{SiO}_{2}$, and $\mathrm{Na}_{2} \mathrm{CaSiO}_{4}$, further promoting the hydration reaction of inorganic soil solidifier components. Yi et al. ${ }^{[4]}$ compared the effect of $\mathrm{NaOH}, \mathrm{Na}_{2} \mathrm{CO}_{3}$ and $\mathrm{Na}_{2} \mathrm{SO}_{4}$ in the solidification of marine soft soil (mineral powder+carbide slag) in Lianyungang Port (in Jiangsu Province) using the principle of alkali excitation, suggesting that three alkali activators can improve the solidifying effect and that $\mathrm{Na}_{2} \mathrm{SO}_{4}$ performed the best.

L. K. Sharma ${ }^{[5]}$ compared the effect of cement and lime added to mountain soil, suggesting that cement had a greater effect on the mechanical properties of soil. The compressive strength of the samples solidified for 28 days was nearly four to six times higher than that of the untreated samples. Through X-ray diffraction (XRD), scanning electron microscopy (SEM), and fourier transform infrared spectroscopy (FTIR) analysis, it is shown that cement and lime can exchange kations with the soil and react with tephros to generate gelling compounds, which ultimately enhances strength.

Nima Latifi et al. ${ }^{[6]}$ studied the solidifying effect of the calcium-based powder solidifier SH-85 on red clay; UCS tests showed that the unconfined compressive strength of soil samples can be increased to 5 times that of the untreated soil after 7 days of solidification. Through the field emission scanning electron microscopy, it is observed that with the extension of the curing time, the surface of the soil particles changed significantly. The solidifier can help to form gelatinous gels and other minerals that can bind soil particles. In addition, they can fill the soil pores, thus making the soil dense and compacted.

The inorganic solidifier generates calcium silicate gel and other minerals mainly through its own hydration reaction. However, when it comes to sulfate soil, the calcium in the solidifier will form ettringite with the aluminum in sulfate soil, causing soil swelling or even destruction. A. Seco ${ }^{[7]}$ et al. used a magnesium-based industrial by-product (PC-8) instead of the calcium-based additive to solidify sulfate rock, suggesting that the magnesium-based solidifier can effectively control soil swelling and that the combination of magnesium-based solidifier and granulated blast furnace slag can deliver even better solidifying effect.

\subsection{Organic soil solidifier}

Organic solidifiers, usually in the form of liquid, can be divided into the sulfonated oil, epoxy resin, modified water glass and polymer material. Its main components can reduce the surface tension of water, thereby thinning the water film layer between soil particles and promoting the ion exchange reaction to replace the kations in the soil. At the same time, the organic soil solidifier usually consists of long-chain polymers, which will entangle with each other and with the soil particles, thereby improving the strength and water stability of the soil. $\mathrm{He}^{[8]}$ treated the loess with a multi-composite soil solidifier of lignin polymer compounds, cement and snail organisms, suggesting that the lignin ( $1 \%$ is the best) can improve the compressive strength and impermeability of the soil. $\mathrm{He}$ also proposed that calcium lignin sulfonate can not only re-arrange the soil particles through the dispersing and coupling effects, but also fill the pores between the soil particles. At the same time, the organic soil solidifier can effectively improve the strength and bearing capacity of the roadbed and is convenient to use. Tao et al. [9] verified the feasibility of using lignin to stabilize silty soil and using the treated soil as a roadbed pavement material, showing that when the lignin content is $12 \%$ and the compaction degree is $96 \%$, the silty soil can deliver excellent mechanical properties. In addition, lignin, as a solidifier for roadbed soil, has a small impact on the environment and low construction costs. Jin et al. ${ }^{[10]}$ studied the effect of organic soil solidifier STW (main component is vinyl acetate) on slope surface clay. Slope scouring experiments showed that STW solidifier can well stabilize the surface layer of slope, on which the vegetation can grow well.

However, organic soil solidifiers are more sensitive to water. Therefore, it is necessary to strictly control the moisture content of the soil when using them. The solidifying effect of organic soil solidifiers may vary greatly in different environments.

\subsection{Ionic soil solidifier}

Ionic soil solidifiers, through the ion exchange with the soil, can reduce the surface tension of water in the soil pores, destroy the capillary film of the soil particles, reduce the gap between the particles, and change the soil from hydrophilic to hydrophobic, making it easier to be compacted. However, as the ion exchange reaction proceeds, the ion concentration of the solution in the soil pores continues to increase until reaching a stable state. The ion concentration of the improved soil, influenced by rain or water migration, will change, thereby weakening the solidification effect. Therefore, ionic soil solidifiers have poor water resistance and are mainly suitable for the 
modification of expansive soils. According to Gouy-Chapman theory, the reduction of the thickness of the electrical layer will reduce the swelling potential of the expansive soil. Therefore, by measuring the kation concentration in the pore water of the expansive soil, we can obtain the value of the swelling potential and evaluate the solidification effect.

$\mathrm{Liu}^{[11]}$ et al. studied the effect of ISS-2500 ionic solidifier on the expansive soil, indicating that the use of ionic solidifier can significantly reduce the expansion rate and water sensitivity of the soil, thus equipping it with good water stability. Similarly, Alhassan et al. ${ }^{[12]}$ studied the solidifying effect of ISS-2500 ionic solidifier through experiments on black cotton soil in northeastern Nigeria. The results showed that this solidifier can change the black cotton soil from alkaline to acidic, thereby promoting the ion exchange reaction. Deng ${ }^{[13]}$ pointed out that the sulfonic group of sulfonated oil (the main component of EN-1 ionic soil solidifier) exchanged ions with the soil particles, thereby changing the hydrophilia of the soil particles; the long-chain hydrocarbyl in the sulfonated oil were interwoven into a net wrapping the soil particles, making them to form together as a whole. Du et al. ${ }^{[14]}$ explored the solidification effect of WH-type soil solidifier on loess canals and soil rainwater collection sites, suggesting that the freezing resistance and impermeability of the loess were greatly improved after solidification.

Ionic solidifiers are commonly used to treat expansive soils, while some inorganic materials can do just as good. Zalihe Nalbantoglu ${ }^{[15]}$ treated the expansive soil using high calcium fly ash (Grade C), which can exchange kations with soil particles and change the thickness of the double electrode layer, thereby effectively reducing the plasticity index and its expansion rate of the soil sample.

\subsection{Biological enzyme soil solidifier}

Biological enzyme soil solidifiers can form a hardened shell on the surface of clay particles by relying on the catalysis effect of biological enzymes and external pressure. Some enzyme components entering the soil will replace the ions with low cohesion, breaking the double electrode layer structure of clay minerals and discharging its moisture, thereby promoting the cementation and accumulation of soil particles and equipping the solidified soil with good integrity and continuity. However, biological enzyme soil solidifiers will slowly degrade over time, and some studies have pointed out that the service life of solidified soil is only about 8 years ${ }^{[16]}$. Commonly used biological enzyme soil solidifiers include Perma-zyme (the most widely used) and TerraZyme. However, there is no clear mainstream theory for the action mechanism of such solidifiers.

Zhang \& $\mathrm{Guo}^{[17]}$ conducted an experimental study on the unconfined compressive strength of fine sand soil, coarse sand soil, sandy loam soil, surface sand soil, and loam clay, pointing out that Perma-zyme can perform better in the soil with more clay particles than in the soil containing more sands and powders in terms of the solidifying effect. Tremblay H. et al. ${ }^{[18]}$ has studied the effect of TerraZyme on soil stabilization for 3 years, showing that this method can reduce road construction costs by $75 \%$, and help to keep the road in good conditions in all four seasons. $\mathrm{Li}^{[19]}$ treated soil samples with TerraZyme, suggesting that the CBR of soil samples could be improved by about $40 \%$.

\subsection{Composite soil solidifier}

It is feasible to combine several types of solidifiers in order to make up for the deficiency of each soil solidifier. The solidifying mechanism of the composite soil solidifier is the synergistic effect and mutual catalytic effect of each solidifier. At present, inorganic solidifiers are used as the main solidifying agent, while organic or ionic agents are used as the auxiliary agent. Wang ${ }^{[20]}$ et al. used ISS and inorganic lime to prepare the composite solidifier, pointing out that ISS can stimulate the activity of lime, accelerate the reaction speed, and improve the soil strength in a short time. For most of the inorganic composite solidifiers, the inorganic material (as the main agent) generates a substance with gelling properties thorough the hydration reaction, while the auxiliary agent stimulates the potential activity of silicon oxide and aluminium oxide in the inorganic material and make them participate in the hydration reaction, thereby further improving the gelatinization and adhesion. In addition, some generated products can fill the pores between the soil particles. Shang et al. ${ }^{[21]}$ used organic and inorganic polymer materials to prepare CHF composite ionic soil solidifier, pointing out that when the solidifier content was $0.02 \%$, the compressive strength and impermeability of the soil can reach the best and at the same time, the free expansion rate can decrease by about $40 \%$. Many hardeners available today need to be combined with inorganic materials such as cement and lime for soil solidification. Yang ${ }^{[22]}$ et al. studied the effects of TG soil solidifier and fiber on the physical-mechanical properties and shrinkage performance of cement-lime soil, showing that when the basalt fiber content is $0.3 \%$ and the TG solidifier is $0.02 \%$, the cement-lime soil can deliver the best performance.

\section{Performance and practical engineering application of soil solidifiers}

\subsection{Solidifying performance}

Three properties needed to be examined after the soil is improved using soil solidifiers, namely, mechanical properties, deformation properties and durability. Different projects in various regions tend to have different emphasis on the performance of soil solidifiers.

\subsubsection{Mechanical properties}

There are four main methods for performing mechanical tests on the improved soil: 1) conduct unconfined 
compressive strength test to measure the compressive strength of the soil body; 2) conduct the splitting strength test to measure the tensile strength; 3 ) use a rebound meter to measure the rebound strength so as to obtain the bending performance; 4) conduct triaxial compression experiment to measure shear resistance.

The soil solidifier content, curing time and soil moisture content have a significant influence on the solidifying effect of soil solidifier. Generally speaking, for the same soil, the larger the amount of soil solidifier, the higher the compressive strength of the improved soil; the compressive strength of the improved soil will continue to increase with the extension of the curing time; there is an optimal value for the initial moisture content, and too high or too low moisture content will adversely affect the compressive strength of the improved soil.

\subsubsection{Deformation performance}

The deformation properties of the soil are divided into deformation by force and volumetric deformation. Deformation by force refers to the tension or compression deformation of the soil when subjected to external forces. Volumetric deformation is the kind of deformation caused by changes in temperature and humidity after solidification, including hygroscopic expansion, drying shrinkage and frozen deformation. The deformation degree is generally measured by linear shrinkage and volume shrinkage.

The soil deformation is not only related to the temperature and humidity of the environment, but also to the nature of the soil itself. Chen et al. ${ }^{[23]}$ pointed out that with the increase of solidifier, the shrinkage limit of salinized soil increased first and then decreased, while the linear shrinkage and volume shrinkage showed the opposite trend.

\subsubsection{Durability}

Durability is closely related to the application and service life of engineering projects. Therefore, the durability of soil solidifier is the basis for the optimization of technical and economic indicators. The durability performance mainly includes the frost resistance, impermeability, and wet-dry cycle resistance of the soil. In some special projects, it is also necessary to examine the scouring resistance and corrosion resistance. For example, the wet-dry cycle resistance test is necessary for channels for seasonal water conveyance, while the corrosion resistance test is required for soils exposed to corrosive environments.

\subsection{Practical engineering applications}

With the continuous development of soil solidifiers, they are now more widely applied in engineering projects, such as water conservancy projects, road projects and special soil treatment.

\subsubsection{Water conservancy projects}

In water conservancy projects, the soil solidifier mainly plays its role in two aspects, namely, channel anti-seepage and slope stabilization.

As for the channel anti-seepage, the soil solidifier mainly focuses on the issues of seepage prevention, anti-scouring, landslide prevention, and sludge solidification. $\mathrm{Li}^{[24]}$ pointed out that the canal water utilization coefficient in China's irrigation area was only about 0.5 , in other words, the amount of water wasted through irrigation channels each year accounted $1 / 3$ of the total water consumption, or $45 \%$ of total agricultural water consumption in China. The channel anti-seepage technique can reduce the leakage loss by $70-90 \%{ }^{[25]}$, thereby increasing the water utilization coefficient of the irrigation channel system and expanding the irrigation area, which is a strong driver for the agricultural production and development in China. Liu et al. ${ }^{[26-27]}$ found that EN-1 solidifier can improve the root vitality of ryegrass, promote its absorption of soil moisture and nutrients; a suitable amount of solidifier can also promote the growth of ryegrass $(0.15 \%$ is the optimal amount). Therefore, soil solidifiers can play a significant role in the improvement of the anti-seepage performance of water conveyance channels. Han ${ }^{[28]}$ et al. used inorganic and organic soil solidifier to solidify the loess, suggesting that the compressive strength and permeability coefficient of the loess were greatly improved after curing, meeting general construction requirements. Zhang et al. ${ }^{[29]}$ used ionic soil solidifier, lime and sand to solidify the sandy silt with high liquid limit in Zhiman Reservoir in Zhanjiang City, showing that the compressive strength, water stability, and impermeability of the improved soil were all satisfactory. Wei et al. ${ }^{[30]}$ carried out an experimental study on the soil and sand in the Ningxia Autonomous Region for the northern climatic environment, showing that the selected solidifier worked well for the sand and gravel, but not for clay. That also reflects the problems of soil solidifiers, including poor adaptability, strong pertinence and poor generality.

Slope solidification, targeting at sandy soil, is a major problem for water conservancy projects. Sand control curing and soil erosion are two important issues for environmental protection ${ }^{[31]}$. Liu et al. ${ }^{[32]}$ used organic polymer soil solidifier to solidify the topsoil of a clay slope in a highway in Jiangsu Province, and planted vegetation on the solidified soil. The results showed that the solidified soil had good water stability and its surface is not washed by rain, and the vegetation can grow well on it. $\mathrm{Su}^{[33]}$ studied the effect of EN-1 solidifier on Pisha sandstone using a combination of natural slope erosion test, indoor geotechnical test, and artificial slope erosion test. The results showed that EN-1 solidifier can improve the ability of the Pisha sandstone slope to resist runoff erosion, reducing soil and water loss; the optimal EN-1 solidifier content shall be $0.2 \%$, the curing time shall be at least $15 \mathrm{~d}$, the compaction shall be controlled above $95 \%$, and the water content shall be controlled to be slightly lower than optimal value. Some scholars ${ }^{[34-35]}$ pointed out that high-molecular polymers can effectively 
inhibit splash erosion and rill erosion, thereby controlling soil erosion. In agriculture, polymers can also prevent the dissolution and loss of fertilizer elements, thereby effectively maintaining the fertilizer efficiency. $\mathrm{Niu}^{[36]}$ proposed a new type of sand consolidation agent, which was composed of potassium water glass solution, new silicon phosphate, lithium silicate solution and silica sol solution, suggesting that this agent can form a binding film on the surface of the sand particles, thereby strengthening the particles, as shown in Figure 1.

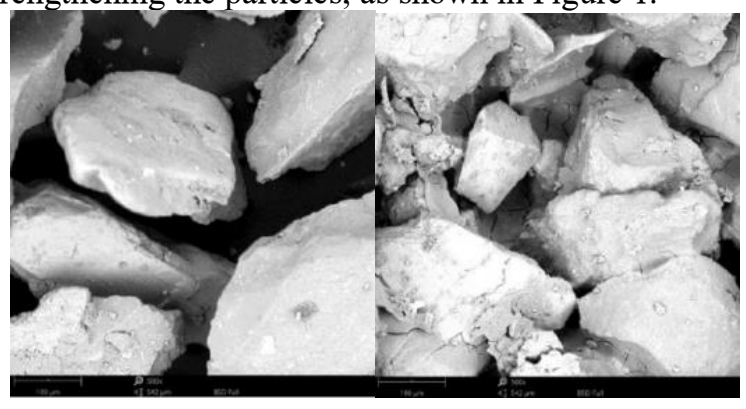

Fig.1. SEM images of sand before solidification (left) and sand after solidification (right)

Zhang $^{[37]}$ compared the effects of CONAID, LUKANG, EN-1 and SSA soil solidifiers on the mechanical properties and erosion resistance of loess, proposing that in loess slope protection projects, the optimal blending amount of EN-1 and LUKANG solidifiers was $0.01 \%$; when the ryegrass planting density was $35 \mathrm{~g} / \mathrm{m} 2$, the compaction degree was $85 \%$, and the age reached $20+$ days, the runoff erosion resistance ability of the slope soil can be significantly improved, thereby preventing slope instability caused by erosion and damage. In addition, this method has little impact on the slope vegetation, thereby realizing soil protection and ecological protection at the same time. $\mathrm{Zhu}^{[38]}$ studied the remediation effect of steel slag fine-powder solidifier on heavy-metal contaminated soil using the orthogonal test. Results showed that after solidifying the soil, the leaching concentrations of $\mathrm{Cr}, \mathrm{Ni}$, $\mathrm{Cu}, \mathrm{Zn}$, and $\mathrm{Pb}$ were reduced by more than $99 \%$; the unconfined compressive strength of the solidified soil can exceed 2.4 MPa, and it had good correlation with time and dosage.

\subsubsection{Road projects}

Soil solidifiers used in road engineering aims to solve the problem of soft soil foundation. They have also been used to deal with environmental issues such as the transportation and landfilling of river and tunnel silt. A lot of free water among soft soil particles leads to the problem of dripping during transportation, causing the problems of road pollution and difficult compaction during landfill.

Wang et al. ${ }^{[39]}$ used ISS solidifier to solidify the subgrade fill of a highway in Qingdao City, Shandong Province, showing that the strength, anti-seepage performance and water stability of the cured soil were greatly improved. $\mathrm{Li}^{[40]}$ used response surface method to study the solidifying effect of self-made solidifier on marine soft soil in Hangzhou City, Zhejiang Province.

Wang et al. ${ }^{[41]}$ conducted research on the subgrade of the temporary airport runway by adding solidifier to the fine sand sample taken in Jiuquan, Gansu Province, showing that $8 \%$ cement plus $0.45 \%$ modified polypropylene fiber can effectively improve the durability against hygroexpansion.

$\mathrm{Shu}^{[42]}$ et al. used modified polypropylene fibers to solidify the sludge, and compared the unconfined compressive strength, moisture content, and shear strength of solidified sludge at different ages. The results showed that the modified polypropylene fibers can effectively improve the physical and mechanical properties of silt soil.

Zhao used cement-lime stabilized lapis as the pavement base in a rural road in Xingtai City, Hebei Province, pointing out that this mixture performed better than lime or cement in terms of stabilization.

$\mathrm{Hu}^{[43]}$ carried out experimental research on a certain section in Tibet with an average hypo of $4500 \mathrm{~m}$ and rainy, snowy, dry, and hot weather conditions using the Perma-zyme soil solidifier. Results showed that the base pavement was neither softened by the rainwater nor spotted with track marks or damages.

\subsubsection{Special soil treatment}

With the development of industrialization, the landfill treatment of various industrial sewage and sludge poses greater difficulty on soil improvement and solidification. Heavy metals will enter the biological chain along with soil water, endangering the ecological environment.

$\mathrm{Xue}^{[44]}$ used three low-cost natural polymer materials, namely lignin (LN), carboxymethyl cellulose, and sodium alginate, to conduct soil improvement trials on the soil contaminated by lead and cadmium in the mining area of Nanjing City. Toxicity Characteristic Leaching Test (TCLP) showed that solidifiers can reduce the bioavailability of lead and cadmium in the soil. The leaching concentrations of lead and cadmium in the treated soil decreased from $5.46 \%$ to $71.1 \%$ and from $4.25 \%$ to $49.6 \%$, respectively, which was because the oxygen-containing groups on the polymer chains can chelate and fix lead and cadmium.

\section{Existing problems and development direction}

Problems have emerged with the wider application of soil solidifiers. A single type of soil solidifier has its own limitations; therefore, soil solidifiers compounded by two or more solidifiers have been continuously promoted. However, the soil quality varies greatly in different regions in China. So the soil solidifier needs to be adjusted for different projects. There is not yet a soil solidifier that can be widely applied. In addition, research mainly focuses on the early-stage performance of solidified soil solidifier, but less on the performance in the later stage.

There is little and limited research on the application of soil solidifiers in alpine regions or the areas exposed to 
special solution and long-term water invasion. Nowadays, for the improvement and solidification of soil, we should consider not only the ways to enhance the mechanical properties, but also the effects on the ecological environment and the issue of soil recovery. Therefore, it is necessary to take into consideration the effects of solidifiers on the heavy metal in the soil, soil $\mathrm{pH}$, and the environmental requirements for plant growth, etc.

The research and development of traditional soil solidifiers requires quantities of experiments, manpower, and material resources. Therefore, we should not simply explore the ways to efficiently develop soil solidifiers through indoor soil mechanics experiments or experiments in the artificially simulated environment; instead, we shall combine computer simulation and experiments. At present, some scholars have investigated the relationship between the components of the solidifier on the soil and the interaction between the components using computer simulations and response surface methodology, and have verified the simulation results through experiments, so that the soil solidifier targeting one specific soil sample can be effectively obtained. Future soil solidifiers should be featured with not only excellent properties and good economic performance, but also good universality and durability.

\section{Reference}

1. Mi gifu, Wang Hao, Liu Jingbing, Yan Hui. Research and Application Progress of Soil Stabilizer[J]. Materials Reports, 2017, 31 (S1):388-391 + 401

2. Gao Jianen, Wu Pute, Lou Zongke. Physicochemical Actions of Stabilized Soil with Cement-based Soil Stabilizer [J]. Rock and Soil Mechanics, 2010,31 (12): 3741-3745

3. HU Mingyu, FU Chao, WEI Lili, ZHENG Jiang. Effect of Inorganic soil stabilizer on properties of raw soil material[J]. Chinese Journal of Materials Research, 2017, 31(06):445-450.

4. YI Yaolin, Li Chen, SUN Chuan, ZHANG Zhengfu, LIU Songyu. Experimental study on alkali induced solidification of lianyungang soft soil [J]. Chinese Journal of rock mechanics and engineering, 2013, 32(09):1820-1826.

5. L.K.Sharma, N.N.Sirdesai, K.M.Sharma, T.N.Singh. Experimental study to examine the independent roles of lime and cement on the stabilization of a mountain soil: A comparative study[J].Applied Clay Science, 2018, 152:183-195

6. Nima Latifi, Amin Eisazadeh, et al.Tropical residual soil stabilization: A powder form material for increasing soil strength[J].Construction and Building Materials, 2017, 147:827-836.

7. A. Seco, L.Miqueleiz, et al. Sulfate soils stabilization with magnesium-based binders[J]. Applied Clay Science, 2017, 135:457-464.

8. He Zhi-qiang. Study on influencing factors and mechanism of reinforced soil with bio-based soil stabilizer [D]. Northwest A\&F University, 2015.

9. Tao Zhang, Guojun Cai, Songyu Liu. Application of lignin-based by-product stabilized silty soil in highway subgrade: A field investigation[J].Journal of Cleaner Production, 2017, 142(4): 4243-4257.

10. Jin Liu, Bin Shi, Hongtao Jiang et al. Research on the stabilization treatment of clay slope topsoil by organic polymer soil stabilizer[J]. Engineering Geology, 2011, 117(1-2): 114-120.

11. Liu qing-bing,xiang wei, zhang wei-feng, cui de-shan. Experimental study of ionic stabilizer-improves expansive soil[J]. Rock and soil mechanics, 2009, 30 (08):2286-2290.

12. Alhassan H M, Olaniyi L F.Effect of'Ionic Soil Stabilizer 2500 'on the properties of black cotton soil[J].British J Appl Sci Technol, 2013, 3(3):406.

13. Deng Weijun. Experimental study on modification of expansive soil by EN-1 ionic soil curing agent [J]. Journal of China \& Foreign Highway, 2015,35 (02):248-250.

14. Du Ying-ji, LI Yuan-ting. Test Research for Characteristics of Solidified Loess [J]. Journal of Irrigation and Drainage,2004(06):65-66+77.

15. Zalihe Nalbantoglu. Effectiveness of Class C fly ash as an expansive soil stabilizer[J]. Construction and Building Materials,2004,18(6):377-381.

16. Fan heng-hui, gao Jian-en, wu pu-te. prospect of Research on soil Stabilizer [J]. Journal of northwest sci-tech univ.of agri.and for.(Nat.Sci.Ed), 2006(02): 141-146+152.

17. Zhang xin-ping, guo liang, peng hong-tao, zhang qi, gu han-lai, su hai-tao. Experimental study on the curing effect of the enzyme on different soils [J]. Building Technology Development, 2009, 36(06): 17-19+56.

18. Tremblay H, Duchesne J, Locat J. Influence of the Nature of Organic Compounds on Fine Soil Stabilization with Cement[J]. Canadian Geotechnical Testing Journal, 2002, 39(3): 535-546.

19. Li Wei. Experimental Study on Soil Solidification by Enzyme Curing Agent [J]. Hunan Communications Science and Technology,2016,42(02):81-85.

20. Wang yiming, Chen yekai, zhang lijuan. development and research of composite soil curing material RBS $[\mathrm{J}]$. Highway traffic science and technology, 2006(05): 107-109.

21. Shang Lu, Wei Dengyuan, Xie Rongwu, Huang Wenyou, Wu Xinxiang, Zhu Guiyu, Qin Guoyi. Study on Soil Mechanical Properties which be Strengthened by Chf Polymer Composite Ion Type Soil Curing Agent [J]. Municipal Engineering Technology,2012,30(S1):159-161.

22. Yang Lin, Liu Yu-tong, Song Yu-xin. Experimental Study on Mechanical Properties and Shrinkage Cement-lime Stabilized Soil Modified with Additives[J]. Journal of China \& Foreign Highway, 2008, 38(01): 288-293.

23. Chen sheng, wang qi, yue yunlong, et al. Preparation 
and properties of high performance soil-Solidified agent [J]. Bulletin of the Chinese Ceramic Society, 2006 (1):109.

24. Li Ruiran, Liu Xu. Research and analysis on the utilization coefficient of irrigation water in typical irrigated areas in China [J]. Journal of shandong agricultural university: natural science, 2012,43 (3):465.

25. He Wuquan, Xing Yichuan, Cai Mingke, Liu Sanhu. New Material and New Technology for Canal Seepage Control and Antifreeze[J]. Water Saving Irrigation, 2003(01):4-5+11-46.

26. Liu Yue-mei, zhang xing-chang, wang dandan. Effects of loess soil stabilization on perenne L.growth and root activity[J].Chinese Journal of Applied Ecology,2011,22(10):2604-2608.

27. Liu Yue-mei, zhang xing-chang. Effects of EN-1 stabilizer, nitrogen fertilizer and soil bulk density on growth and water use efficiency of ryegrass [J]. Journal of Northwest A \& F University (Natural Science Edition),2014,42(1): 151-158.

28. Han Sujian, Guo Minxia, Li Ning. Discussion on Soil Stabilizer for Seepage Control of Canal in Loess Area [J]. Bulletin of Soil and Water Conservation,2005(02):60-62.

29. Zhang liJuan, Wang yiming, Chen Yekai, et al.Experimental Study on the Application of Soil Stabilizer ISS in Canal Seepage Control[J]. China Rural Water and Hydropower, 2004(6): 18 .

30. Wei wei, Peng jun, Zhang hua.Discussion and application test of soil solidifier in irrigation work[J].Ningxia Engineering Technology, 2004,3 (2) $: 174$

31. Zhang Guanhua, Niu Jun, Sun Jinwei, Li Hao. Research progress of soil curing agent and its application in soil and water conservation [J]. Soils, 2008,50(01):28-34.

32. Liu J, Shi B, Jiang $H$, et al.Research on the stabilization treatment of clay slope topsoil by organic polymer soil stabilizer[J].Eng Geology, 2011, 117(1-2):114.

33. Su Tao. Mechanism on scourresistance stability of en-1 solidified slope in pisha sandstone
region[D].Northwest A \& F University, 2011.

34. I.SHAINBERG, G.J. LEVY, P. RENGASAMY, H.FRENKEL. AGGREGATE STABILITY AND SEAL FORMATION AS AFFECTED BY DROPS' IMPACT ENERGY AND SOIL AMENDMENTS[J]. Soil Science, 1992, 154(2).

35. Yan Rong, Li Yundong, Fang Yanming, Yang Linzhang, wu yong-hong. Effect of Soil Ameliorants on Phosphorus Loss [J]. Soils,2011,43(02):203-209.

36. Niu Xiaodi. Curing Mechanism and Experimental Study of Sandy Slope[D]. Shijiazhuang TIEDAO university, 2017.

37. Zhang Liping. Researches on Loess Slope Stability and Prevention Strategies[D].Northwest A \& F University,2009.

38. Zhu Lijun, Wang Lei, Cheng Dongbo, Liu Guowui, Long Tao, Jin Qiang. Experimental Study on the Application of Steel Slag Powder in Soil Stabilizer Contaminated by Heavy Metals [J].Bulletin of the Chinese Ceramic Society,2016,35(07):2281-2286.

39. Wang Jiatao, Zhou Jian. Experimental and Study on Subgrade Soil Modified with Ioil Soil Stabilizer [J]. Highway, 2010(12):79-82.

40. Li Xuegang. Theoretical study on stabilization of marine soft clay in Hangzhou [D]. Zhejiang university,2013.

41. Wang tian, weng xingzhong, zhang jun, jiang le, liu pengcheng, zhang shi. Strength characteristics of fiber-reinforced sand under dry-wet cycles[J].Journal of Railway Science and Engineering,2017,14(04):721-729.

42. Shu Lin Zhan,Shu Sen Gao,Jun Ying Lai.Research about the effect of Polypropylene Fiber on mechanical properties of curing sludge [J].Advanced Materials Research, 2011, 250-253:788-794.

43. Hu Shiquan. On the application of Parma road construction technology in Lhasa $[\mathrm{J}]$. Highway traffic technology (applied technology edition),2007(03):78-81.

44. Xue Tao,Aimin $\mathrm{Li}, \mathrm{Hu}$ Yang.Immobilization of metals in contaminated soils using natural polymer-based stabilizers[J].Environmental Pollution,2017,222:348-355. 